\title{
Profit maximization and supermodular technology
}

\author{
Christopher P. Chambers • Federico Echenique
}

Received: 5 December 2006 / Accepted: 1 February 2008 / Published online: 26 February 2008 (C) Springer-Verlag 2008

\begin{abstract}
A dataset is a list of observed factor inputs and prices for a technology; profits and production levels are unobserved. We obtain necessary and sufficient conditions for a dataset to be consistent with profit maximization under a monotone and concave revenue based on the notion of cyclic monotonicity. Our result implies that monotonicity and concavity cannot be tested, and that one cannot decide if a firm is competitive based on factor demands. We also introduce a condition, cyclic supermodularity, which is both necessary and sufficient for data to be consistent with a supermodular technology. Cyclic supermodularity provides a test for complementarity of production factors.
\end{abstract}

Keywords Complementarity - Afriat's theorem - Factor demands ·

Revealed preference

\section{JEL Classification D21 D D24}

\section{Introduction}

We study the testable implications of monotone and supermodular revenues and technologies. We suppose that we are given data on a firm's factor demands at various factor prices, but that profits and output are unobservable. We show that the data are

\footnotetext{
We are very grateful to two anonymous referees for suggestions, comments, and corrections. We also thank Kim Border for his suggestions on an earlier draft.

C. P. Chambers · F. Echenique ( $\square)$

Division of the Humanities and Social Sciences, California Institute of Technology,

Pasadena, CA 91125, USA

e-mail: fede@hss.caltech.edu

C. P. Chambers

e-mail: chambers@hss.caltech.edu
} 
consistent with profit maximization if and only if they are consistent with a concave and monotone technology. Moreover, the data are rational if and only if they are "cyclically monotone." Consequently, monotonicity and concavity have no testable implications in this environment. Second, we provide a condition characterizing datasets which could be generated by a technology with complementary production factors, where complementarity means that the technology is supermodular. The condition is a strengthening of the one we have obtained for monotonicity. While it may not be easy to verify whether the condition is satisfied in general, it is usually quite simple to refute. Moreover, in many special cases, the condition is in fact easy to verify.

That monotonicity is not testable is potentially interesting because it implies that one cannot test if a firm is competitive based on factor demands. To some extent, the result is a formalization of the notion that a profit-maximizing firm will not operate where its revenues are monotone decreasing.

Our research follows Sydney Afriat's $(1967,1972)$ seminal papers in studying the testable implications of maximizing behavior. Afriat studied the refutability of concave and monotone production functions. Afriat assumes that both factor demands and production output are observable. When output is observable, one can directly test if the output is supermodular in the factors used. We assume instead that only factors and their prices are observed, and we ask when these can be rationalized using a supermodular technology. Our assumptions make sense when studying, for example, household production (Becker 1965; Lancaster 1966), or other areas where one may observe factor demands but not outputs.

Other early papers on testing assumptions on technologies are Hanoch and Rothschild (1972), Diewert and Parkan (1983) and Varian (1984). Like Afriat, they assume data on output, not purely on factor demands. They do not test for complementarities in production (supermodularity). Varian (1984) discusses how one can use his results to test for non-competitive behavior by a firm. But his results are positive, in the sense that it is possible to test anti-competitive behavior.

Our theorem relies on basic duality results from linear programming and convex analysis, and in this sense rests on ideas similar to those found in Fostel et al. (2004) and Chung-Piaw and Vohra (2003) (see also Fishburn 1971).

A paper similar in spirit and results to ours is Brown and Calsamiglia (2007). They study problems of consumer behavior, obtaining necessary and sufficient conditions for demand data to be rationalizable by a quasilinear utility function. Their condition is a list of inequalities strengthening Afriat's; but they also provide a cyclic monotonicity condition that demand data must satisfy. They do not study supermodularity, however.

In Chambers and Echenique (2006), we studied the testable implications of supermodularity in ordinal economic models. The model we are considering here is not ordinal, and the results of the two papers are independent.

We present definitions and background results in Sect. 2. We present the results in Sect. 3.

\section{Preliminary definitions and results}

We use $\leq$ to denote the usual partial order on $\mathbb{R}^{n}$. A set $X \subseteq \mathbb{R}^{n}$ is a lattice if, for all $x, y \in X$, there exists a unique greatest lower bound $x \wedge y$ and a unique 
least upper bound $x \vee y$ in $X$ according to $\leq$. We write $x \| y$ if neither $x \leq y$ or $y \leq x$.

For an arbitrary set $X$, say that a function $u: X \rightarrow \mathbb{R}$ is monotone increasing if for all $x, y \in X, x \leq y$ implies $u(x) \leq u(y)$. A function $u: X \rightarrow \mathbb{R}$ is supermodular (see Topkis 1998; Vives 1999) if, for all $x, y \in X, u(x \vee y)+u(x \wedge y) \geq u(x)+$ $u(y)$.

\section{Results}

We imagine that we observe a finite set of price and factor input data. Prices are elements of $\mathbb{R}_{+}^{n}$, and factor input data are elements of some finite $X \subseteq \mathbb{R}_{+}^{n}$. We denote by $w \in \mathbb{R}_{+}^{n}$ a vector of factor prices and $z \in X$ is a vector of factor demands at prices $w$. Thus, an observation is a pair $(w, z) \in \mathbb{R}_{+}^{n} \times X$.

Let $X \subseteq Y$. We say that a function $f: Y \rightarrow \mathbb{R}$ rationalizes a finite set of observations $\left\{\left(w_{k}, z_{k}\right)\right\}_{k=1}^{K}$ if, for all $k=1, \ldots, K$ and all $z \in Y$,

$$
f\left(z_{k}\right)-w_{k} \cdot z_{k} \geq f(z)-w_{k} \cdot z
$$

Interpret $f(z)$ as the revenue the firm receives when it uses factors $z$.

If the firm in question is competitive, $f(z)$ is proportional to its production function. So our results can be interpreted as statements about revenue, or about technology, if one makes the assumption that the firm is competitive.

Proposition 1 Given is a finite set of observations $\left\{\left(w_{k}, z_{k}\right)\right\}_{k=1}^{K}$. The following statements are equivalent.

(a) There exists $f: \bigcup_{k=1}^{K}\left\{z_{k}\right\} \rightarrow \mathbb{R}$ rationalizing the observations.

(b) There exists a concave and monotone $f: \mathbb{R}_{+}^{n} \rightarrow \mathbb{R}$ rationalizing the observations.

(c) For all finite sequences $\left\{\left(w^{i}, z^{i}\right)\right\}_{i=1}^{L} \subseteq\left\{\left(w_{k}, z_{k}\right)\right\}_{k=1}^{K}$,

$$
\sum_{i=1}^{L} w^{i} \cdot\left(z^{i}-z^{i+1}\right) \leq 0(\text { addition here is } \bmod L) .
$$

Proof We establish that $\mathrm{a} \Longrightarrow \mathrm{c} \Longrightarrow \mathrm{b} \Longrightarrow \mathrm{a}$.

The implication $\mathrm{b} \Longrightarrow \mathrm{a}$ is trivial. To see that $\mathrm{a} \Longrightarrow \mathrm{c}$, let $f$ rationalize the observations, and let $\left\{\left(w^{i}, z^{i}\right)\right\}_{i=1}^{L} \subseteq\left\{\left(w_{k}, z_{k}\right)\right\}_{k=1}^{K}$. In particular,

$$
f\left(z^{i}\right)-w^{i} \cdot z^{i} \geq f\left(z^{i+1}\right)-w^{i} \cdot z^{i+1}
$$

Consequently,

$$
f\left(z^{i}\right)-f\left(z^{i+1}\right) \geq w^{i} \cdot\left(z^{i}-z^{i+1}\right) .
$$


Thus

$$
\sum_{i=1}^{L}\left[f\left(z^{i}\right)-f\left(z^{i+1}\right)\right] \geq \sum_{i=1}^{L}\left[w^{i} \cdot\left(z^{i}-z^{i+1}\right)\right] .
$$

However, the left hand side of this inequality is zero. Thus, $\sum_{i=1}^{L} w^{i} \cdot\left(z^{i}-\right.$ $\left.z^{i+1}\right) \leq 0$.

Lastly, consider the correspondence $\rho: \mathbb{R}_{+}^{n} \rightrightarrows \mathbb{R}_{+}^{n}$ defined by $\rho(z)=\{w:(w, z) \in$ $\left.\left\{\left(w_{k}, z_{k}\right)\right\}_{k=1}^{K}\right\}$. Condition (b) states that this correspondence is cyclically monotone. By Theorem 24.8 of Rockafellar (1970), there exists $f: \mathbb{R}_{+}^{n} \rightarrow \mathbb{R}$ which is concave for which for all $\left(w_{i}, z_{i}\right) \in\left\{\left(w_{k}, z_{k}\right)\right\}_{k=1}^{K}$ and all $z \in \mathbb{R}_{+}^{n}, f\left(z_{i}\right)+w_{i} \cdot\left(z-z_{i}\right) \geq f(z)$. Consequently, $f\left(z_{i}\right)-w_{i} \cdot z_{i} \geq f(z)-w_{i} \cdot z$ for all $z \in \mathbb{R}_{+}^{n}$. That $f$ is monotonic follows from the construction in Rockafellar and the fact that $w_{i} \geq 0$ for all $i$.

Proposition 1 is the direct consequence of a result in convex analysis (Rockafellar 1970). We are not the first to draw attention to this result in the economics literature: Brown and Calsamiglia (2007) present a similar application. They characterize, in a demand context, those price and consumption bundle observations which can be generated by the maximization of a quasilinear utility function. They obtain a necessary and sufficient condition which is effectively our condition (c).

Our second result tries to understand the implications of a supermodular revenue function. Supermodularity in this context can be interpreted as complementarity of inputs (Topkis 1998; Vives 1999). Here, we will suppose $X \subseteq \mathbb{R}_{+}^{n}$ is a finite lattice according to $\leq$. We will provide a necessary and sufficient condition that a set of data must satisfy in order to be rationalizable by a supermodular $f$.

Before we begin, we start with some preliminaries. We first define three binary relations on $X$. Fix a set of data $\left\{\left(w_{k}, z_{k}\right)\right\}_{k=1}^{K}$. We define for all $k=1, \ldots, K$ and for all $z \in X, z_{k} T z{ }^{1}$ That is, every observed demand beats every other possible demand with $T$. For $z, z^{\prime} \in X$, define $z U z^{\prime}$ if there exists $x \| z^{\prime}$ such that $z=x \vee z^{\prime}$. Define $z D z^{\prime}$ if there exists $x \| z^{\prime}$ such that $z=x \wedge z^{\prime}$. The relations $U$ and $D$ can be viewed as jumps "up" and "down" in terms of $\leq$. Note that $(z \vee x, z) \in U$ if and only if $(z \wedge x, x) \in D$.

There may be more than one reason for $z R z^{\prime}, R \in\{T, U, D\}$. We use a notation that keeps track of more than just the pair $z$ and $z^{\prime}$. When $z T z^{\prime}$, we keep track of the salary $\lambda=w_{k}$ at which $z=z_{k}$ was demanded. When $z U z^{\prime}$ or $z D z^{\prime}$, we keep track of the $x$ for which $z=x \vee z^{\prime}$ or $z=x \wedge z^{\prime}$, respectively.

The notation we use is as follows. When $z T z^{\prime}$ we write the expression

$$
\left(\left(z, z^{\prime}\right), T, \lambda, y\right)
$$

which has $\lambda=w_{k}$ for a $k$ with $z=z_{k}$, and $y=z^{\prime}$. When $z U z^{\prime}$ we write $\left(\left(z, z^{\prime}\right)\right.$, $U, \lambda, x)$, which has $\lambda=0$ and $x \vee z^{\prime}=z$. Symmetrically, when $z D z^{\prime}$ we write the expression $\left(\left(z, z^{\prime}\right), D, \lambda, x\right)$, which has $\lambda=0$ and $x \wedge z^{\prime}=z$.

\footnotetext{
1 This relation clearly depends on the dataset in question; however, in the interest of notational simplicity, we suppress this dependence.
} 
A cycle according to $(T, U, D)$ (or simply a $(T, U, D)$ cycle) is a finite set $\left\{\left(z^{i}, z^{i+1}\right), R^{i}, \lambda^{i}, x^{i}\right\}_{i=1}^{L}$ for which for all $i=1, \ldots, L$, the expression

$$
\left(\left(z^{i}, z^{i+1}\right), R^{i}, \lambda^{i}, x^{i}\right)
$$

is true (addition here is $\bmod L) .^{2}$ Denote the set of all $(T, U, D)$ cycles by $\varrho$.

Here is our primary requirement:

Cyclic supermodularity: Let $n \in \mathbb{Z}_{+}^{\varrho}$ for which for all ordered pairs $(x, y) \in X^{2}$ such that $x \| y$,

$$
\sum_{\{\rho \in \varrho:((x \vee y, x), U, 0, y) \in \rho\}} n_{\rho}=\sum_{\{\rho \in \varrho:((x \wedge y, y), D, 0, x) \in \rho\}} n_{\rho} .
$$

Then

$$
\sum_{\rho \in \varrho} n_{\rho} \sum_{\left(\left(z, z^{\prime}\right), T, \lambda, z^{\prime}\right) \in \rho} \lambda \cdot\left(z-z^{\prime}\right) \leq 0 .
$$

In particular, note that cyclic supermodularity is a strengthening of condition (c) in Proposition 1.

Proposition 2 A set of data $\left\{\left(w_{k}, z_{k}\right)\right\}_{k=1}^{K}$ can be rationalized by a supermodular $f: X \rightarrow \mathbb{R}$ if and only if it is cyclically supermodular.

The proof appears in an Appendix. Proposition 2 obtains the complete testable implications for the rationalization of data by a supermodular production function. Verifying that cyclic supermodularity is satisfied can be very difficult, but the condition often provides an easy method of verifying when a set of data does not have a supermodular rationalization. An example follows:

Example 1 Let $X=\{0,1\}^{2}$. Suppose that we have two demand observations, one at prices $w_{1}=(0,0)$ and the other at prices $w_{2}=(1,1)$. At $w_{1}, z_{1}=(0,1)$ is demanded, whereas at $w_{2}, z_{2}=(1,0)$ is demanded. Consequently, any $f$ which rationalizes the data must satisfy

$$
\begin{array}{r}
f(0,1) \geq f(1,1) \text { by observation }\left(w_{1}, z_{1}\right) \\
f(1,0)-1 \geq f(0,0) \text { by observation }\left(w_{2}, z_{2}\right) .
\end{array}
$$

Summing the two inequalities obtains $f(0,1)+f(1,0)-1 \geq f(1,1)+f(0,0)$, so that

$$
f(0,1)+f(1,0)>f(1,1)+f(0,0),
$$

\footnotetext{
2 Note that two distinct cycles according $(T, U, D)$ may be the same cycles according to $T \cup U \cup D$. This is because there may be some overlap between the three relations $T, U$, and $D$.
} 
a direct contradiction to supermodularity. Nevertheless, the function $f$ defined by

$$
\begin{aligned}
& f(0,0)=0 \\
& f(0,1)=1 \\
& f(1,0)=1 \\
& f(1,1)=1
\end{aligned}
$$

is easily seen to rationalize the data. Our necessary and sufficient condition is easily seen to be violated by considering the $(T, U, D)$-cycle

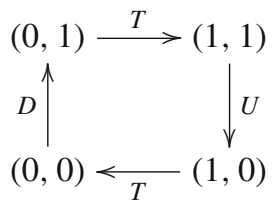

Formally:

$$
\begin{aligned}
& (((1,0),(0,0)), T,(1,1),(0,0)) \\
& (((0,0),(0,1)), D,(0,0),(1,0)) \\
& (((0,1),(1,1)), T,(0,0),(1,1)) \\
& (((1,1),(1,0)), U,(0,0),(0,1))
\end{aligned}
$$

and

$$
(1,1) \cdot((1,0)-(0,0))+(0,0) \cdot((0,1)-(1,1))>0,
$$

a contradiction to cyclic supermodularity.

Finally, we present a simple corollary with a sufficient condition for the existence of a supermodular rationalization.

Corollary 1 If for all $\rho \in \varrho$, we have $\sum_{\left(\left(z, z^{\prime}\right), T, \lambda, z^{\prime}\right) \in \rho} \lambda \cdot\left(z-z^{\prime}\right) \leq 0$, then there exists a supermodular $f: X \rightarrow \mathbb{R}$ rationalizing the data.

\section{Appendix: Proof of Proposition 2}

Let us first suppose that the set of data $\left\{\left(w_{k}, z_{k}\right)\right\}_{k=1}^{K}$ can be rationalized by a supermodular $f: X \rightarrow \mathbb{R}$. Let $n \in \mathbb{Z}_{+}^{\varrho}$ satisfy Condition (1) for all $x \| y$. Since $f$ is supermodular, $f(x \vee y)-f(x)+f(x \wedge y)-f(y) \geq 0$. So Condition (1) implies 
that, for all $x, y \in X$ such that $x \| y$,

$$
\begin{gathered}
\sum_{\{\rho \in \varrho:((x \vee y, x), U, 0, y) \in \rho\}} n_{\rho}[f(x \vee y)-f(x)] \\
+\sum_{\{\rho \in \varrho:((x \wedge y, y), D, 0, x) \in \rho\}} n_{\rho}[f(x \wedge y)-f(y)] \geq 0 .
\end{gathered}
$$

Conclude that, by summing over all ordered pairs $(x, y)$ for which $x \| y$,

$$
\begin{aligned}
& \sum_{\rho \in \varrho} n_{\rho}\left[\left(\sum_{\left(\left(z \vee z^{\prime}, z^{\prime}\right), U, 0, z\right) \in \rho} f\left(z \vee z^{\prime}\right)-f\left(z^{\prime}\right)\right)\right. \\
& \left.+\left(\sum_{\left(\left(z \wedge z^{\prime}, z\right), D, 0, z^{\prime}\right) \in \rho} f\left(z \wedge z^{\prime}\right)-f(z)\right)\right] \geq 0 .
\end{aligned}
$$

Now, since each $\rho \in \varrho$ is a cycle, we have

$$
\sum_{\left(\left(z, z^{\prime}\right), R, \lambda, x\right) \in \rho} n_{\rho}\left[f(z)-f\left(z^{\prime}\right)\right]=0
$$

Conclude

$$
\begin{aligned}
0= & \sum_{\rho \in \varrho} n_{\rho} \sum_{\left(\left(z, z^{\prime}\right), R, \lambda, x\right) \in \rho}\left[f(z)-f\left(z^{\prime}\right)\right] \\
= & \sum_{\rho \in \varrho} n_{\rho} \sum_{\left(\left(z, z^{\prime}\right), T, \lambda, z^{\prime}\right) \in \rho}\left[f(z)-f\left(z^{\prime}\right)\right] \\
& +\sum_{\rho \in \varrho} n_{\rho}\left[\begin{array}{c}
\left(\sum_{\left(\left(z \vee z^{\prime}, z^{\prime}\right), U, 0, z\right) \in \rho} f\left(z \vee z^{\prime}\right)-f\left(z^{\prime}\right)\right) \\
+\left(\sum_{\left(\left(z \wedge z^{\prime}, z\right), D, 0, z^{\prime}\right) \in \rho} f\left(z \wedge z^{\prime}\right)-f\left(z^{\prime}\right)\right)
\end{array}\right] \\
\geq & \sum_{\rho \in \varrho} n_{\rho} \sum_{\left(\left(z, z^{\prime}\right), T, \lambda, z^{\prime}\right) \in \rho}\left[f(z)-f\left(z^{\prime}\right)\right] .
\end{aligned}
$$

Since $f$ rationalizes the data, for all $\left(\left(z, z^{\prime}\right), T, \lambda, z^{\prime}\right) \in T, f(z)-f\left(z^{\prime}\right) \geq \lambda \cdot\left(z-z^{\prime}\right)$. Therefore,

$$
\sum_{\rho \in \varrho} n_{\rho} \sum_{\left(\left(z, z^{\prime}\right), T, \lambda, z^{\prime}\right) \in \rho}\left[f(z)-f\left(z^{\prime}\right)\right] \geq \sum_{\rho \in \varrho} n_{\rho} \sum_{\left(\left(z, z^{\prime}\right), T, \lambda, z^{\prime}\right) \in \rho} \lambda \cdot\left(z-z^{\prime}\right) .
$$

Therefore,

$$
\sum_{\rho \in \varrho} n_{\rho} \sum_{\left(\left(z, z^{\prime}\right), T, \lambda, z^{\prime}\right) \in \rho} \lambda \cdot\left(z-z^{\prime}\right) \leq 0
$$


so that cyclic supermodularity is satisfied.

We will now show that if there does not exist a supermodular rationalizing function, then cyclic supermodularity is violated. The existence of a supermodular rationalizing function is equivalent to the existence of a vector $u \in \mathbb{R}^{X}$ satisfying the following conditions. For all $z T z^{\prime}$ and all $k$ for which $z=z_{k},\left(1_{z}-1_{z^{\prime}}\right) \cdot u \geq w_{k} \cdot\left(z-z^{\prime}\right)$, and for all ordered pairs $\left(z, z^{\prime}\right) \in X^{2}$ with $z \| z^{\prime},\left(1_{z \vee z^{\prime}}+1_{z \wedge z^{\prime}}-1_{z}-1_{z^{\prime}}\right) \cdot u \geq 0$.

We will show that if such a vector does not exist, then cyclic supermodularity is violated. In particular, by Theorem 22.1 in Rockafellar (1970), if such a vector does not exist, there exists for all ordered pairs $\left(z, z^{\prime}\right) \in T$ and all $k$ for which $z=z_{k}$, a nonnegative number $\eta_{\left(\left(z, z^{\prime}\right), T, w_{k},\left(z, z^{\prime}\right)\right)}$, and for all unordered pairs $z, z^{\prime} \in X$ such that $z \| z^{\prime}$, a nonnegative number $\alpha_{\left\{z, z^{\prime}\right\}}$ such that

$$
\begin{aligned}
& \sum_{\left(\left(z, z^{\prime}\right), T, \lambda, z^{\prime}\right)} \eta_{\left(\left(z, z^{\prime}\right), T, \lambda,\left(z, z^{\prime}\right)\right)}\left(1_{z}-1_{z^{\prime}}\right) \\
& +\sum_{\substack{z, z^{\prime} \in X \\
z \| z^{\prime}}} \alpha_{\left\{z, z^{\prime}\right\}}\left(1_{z \vee z^{\prime}}+1_{z \wedge z^{\prime}}-1_{z}-1_{z^{\prime}}\right)=0
\end{aligned}
$$

and

$$
\sum_{\left(\left(z, z^{\prime}\right), T, \lambda,\left(z, z^{\prime}\right)\right)} \eta_{\left(\left(z, z^{\prime}\right), T, \lambda,\left(z, z^{\prime}\right)\right)} \lambda \cdot\left(z-z^{\prime}\right)>0 .
$$

Note that the 0 in (2) is the null vector in $\mathbb{R}^{X}$.

We now use the numbers $\alpha_{\left\{z, z^{\prime}\right\}}$ to construct the weights $\eta_{\left(\left(z, z^{\prime}\right), R, \lambda,(x, y)\right)}$ when $R \neq T$. For all unordered pairs $z, z^{\prime}$ for which $z \| z^{\prime}$, choose nonnegative numbers $\eta_{\left(\left(z \vee z^{\prime}, z\right), U, 0, z^{\prime}\right)}$ and $\eta_{\left(\left(z \wedge z^{\prime}, z^{\prime}\right), D, 0, z^{\prime}\right)}$ by fixing ordering $z, z^{\prime}$ arbitrarily and choosing

$$
\begin{aligned}
& \eta_{\left(\left(z \vee z^{\prime}, z\right), U, 0, z^{\prime}\right)}=\eta_{\left(\left(z \wedge z^{\prime}, z^{\prime}\right), D, 0, z\right)}=\alpha_{\left\{z, z^{\prime}\right\}} . \\
& \eta_{\left(\left(z \vee z^{\prime}, z^{\prime}\right), U, 0, z\right)}=\eta_{\left(\left(z \wedge z^{\prime}, z\right), D, 0, z^{\prime}\right)}=0 .
\end{aligned}
$$

Then the sum in (2) results in:

$$
\sum_{\left(\left(z, z^{\prime}\right), R, \lambda, x\right)} \eta_{\left(\left(z, z^{\prime}\right), R, \lambda, x\right)}\left(1_{z}-1_{z^{\prime}}\right)=0 .
$$

So the nonexistence of a supermodular rationalization implies (4) and (3).

We claim that there is a vector $\left(y_{\rho}\right)_{\rho \in \varrho} \in \mathbb{R}_{+}^{\varrho}$ such that

$$
\eta_{\left(\left(z, z^{\prime}\right), R, \lambda,(x, y)\right)}=\sum_{\left\{\rho \in \varrho:\left(\left(z, z^{\prime}\right), R, \lambda,(x, y)\right) \in \rho\right\}} y_{\rho} .
$$

The argument is similar to that of the argument establishing the Theorem of Poincaré-Veblen-Alexander (see Berge 2001, Chap. 15, Theorem 5), so we only sketch it here. If $\eta_{\left(\left(z, z^{\prime}\right), R, \lambda, x\right)}=0$ for all possible elements of a $(T, U, D)$ 
cycle, we are done (by choosing $y_{\rho}=0$ for all $(T, U, D)$ cycles). (Of course, as $\sum_{\left(\left(z, z^{\prime}\right), T, \lambda, z^{\prime}\right)} \eta_{\left(\left(z, z^{\prime}\right), T, \lambda, z^{\prime}\right)} \lambda \cdot\left(z-z^{\prime}\right)>0$, this is impossible). So, fix some $\left(\left(z, z^{\prime}\right), R, \lambda, x\right)$ for which $\eta_{\left(\left(z, z^{\prime}\right), R, \lambda, x\right)}>0$. By equality (4), there exists some $z^{\prime \prime}$ such that $\eta_{\left(\left(z^{\prime}, z^{\prime \prime}\right), R, \lambda^{\prime}, x^{\prime}\right)}>0$ for some $\lambda^{\prime}, x^{\prime}$. Repeat the procedure with $z^{\prime \prime}$, finding a corresponding $z^{\prime \prime \prime}$. Since the set $X$ is finite, eventually these elements will form a $(T, U, D)$ cycle $\rho$ for which, for all $\left(\left(z, z^{\prime}\right), R, \lambda, x\right) \in \rho, \eta_{\left(\left(z, z^{\prime}\right), R, \lambda, x\right)}>0$. Set $y_{\rho}=\inf _{\left(\left(z, z^{\prime}\right), R, \lambda, x\right) \in \rho} \eta_{\left(\left(z, z^{\prime}\right), R, \lambda, x\right)}>0$. We now obtain a new vector $\eta^{\prime}$ indexed by the elements of a $(T, U, D)$ cycle, defined as $\eta_{\left(\left(z, z^{\prime}\right), R, \lambda, x\right)}^{\prime}=\eta_{\left(\left(z, z^{\prime}\right), R, \lambda, x\right)}-$ $y_{\rho} 1_{\left(\left(z, z^{\prime}\right), R, \lambda, x,\right) \in \rho} \geq 0$. Moreover, $\eta^{\prime}$ has at least one more zero element than $\eta$ and also satisfies an equality such as (4). We therefore repeat the procedure with this $\eta^{\prime}$, eventually obtaining (5) after a finite number of steps.

Therefore,

$$
\sum_{\left(\left(z, z^{\prime}\right), R, \lambda, x\right)} \sum_{\left\{\rho \in \varrho:\left(\left(z, z^{\prime}\right), R, \lambda, x\right) \in \rho\right\}} y_{\rho}\left(1_{z}-1_{z^{\prime}}\right)=0
$$

and

$$
\sum_{\left(\left(z, z^{\prime}\right), T, \lambda, z^{\prime}\right)} \sum_{\left\{\rho \in \varrho:\left(\left(z, z^{\prime}\right), T, \lambda, z^{\prime} \in \rho\right\}\right.} y_{\rho} \lambda \cdot\left(z-z^{\prime}\right)>0 .
$$

Equivalently,

$$
\sum_{\rho \in \varrho} y_{\rho} \sum_{\left\{\left(z, z^{\prime}\right):\left(\left(z, z^{\prime}\right), R, \lambda, x\right) \in \rho\right\}}\left(1_{z}-1_{z^{\prime}}\right)=0
$$

and

$$
\sum_{\rho \in \varrho} y_{\rho} \sum_{\left(\left(z, z^{\prime}\right), T, \lambda, z^{\prime}\right) \in \rho} \lambda \cdot\left(z-z^{\prime}\right)>0 .
$$

Moreover, for all $z, z^{\prime}$ for which $z \| z^{\prime}$,

$$
\eta_{\left(\left(z \vee z^{\prime}, z\right), U, 0, z, z^{\prime}\right)}=\eta_{\left(\left(z \wedge z^{\prime}, z^{\prime}\right), D, 0, z\right)}
$$

so that

$$
\sum_{\left(\left(z \vee z^{\prime}, z\right), U, 0, z^{\prime}\right) \in \rho} y_{\rho}=\sum_{\left(\left(z \wedge z^{\prime}, z^{\prime}\right), D, 0, z\right) \in \rho} y_{\rho} .
$$

We now claim that we may without loss of generality choose each $y_{\rho}$ to be integer valued. For any collection of rational vectors $p_{1}, \ldots, p_{M} \in \mathbb{Q}^{\varrho}$, there is a set of rational vectors forming a basis for $\left\{x \in \mathbb{R}^{\varrho}: x \cdot p_{i}=0\right.$ for all $\left.i=1, \ldots, M\right\}$, consequently rational vectors are dense in $\left\{x \in \mathbb{R}^{\varrho}: x \cdot p_{i}=0\right.$ for all $\left.i=1, \ldots, M\right\}$. 
Note that, viewed as a vector in $\mathbb{R}^{\varrho}$,

$$
q^{*}=\left(\sum_{\left\{\left(z, z^{\prime}\right):\left(\left(z, z^{\prime}\right), R, \lambda, x \in \rho\right\}\right.}\left(1_{z}-1_{z^{\prime}}\right)\right)_{\rho \in \varrho} \in \mathbb{Q}^{\varrho},
$$

so that $y \cdot q^{*}=0$. Moreover, for all ordered pairs $z, z^{\prime} \in X$ for which $z \| z^{\prime}$,

$$
q_{z, z^{\prime}}=\left(1_{\left(\left(z \vee z^{\prime}, z\right), U, 0, z^{\prime}\right) \in \rho}-1_{\left(\left(z \wedge z^{\prime}, z^{\prime}\right), D, 0, z\right) \in \rho}\right)_{\rho \in \varrho} \in \mathbb{Q}^{\rho} .
$$

Note that $y \cdot q_{z, z^{\prime}}=0$ as well. Lastly, for all $\rho \in \varrho$ for which $y_{\rho}=0$, we know that $y \cdot 1_{\rho}=0$. There is a rational vector $y^{\prime}$ satisfying all of these equalities which is arbitrarily close to $y$; consequently we may choose $y^{\prime} \in \mathbb{Q}_{+}^{Q}$ such that $\sum_{\rho \in \varrho} y_{\rho}^{\prime} \sum_{\left(\left(z, z^{\prime}\right), T, \lambda, z^{\prime}\right) \in \rho} \lambda \cdot\left(z-z^{\prime}\right)>0$. Note that $y_{\rho}=0 \Longrightarrow y_{\rho}^{\prime}=0$ and that

$$
\sum_{\left(\left(z \vee z^{\prime}, z\right), U, 0, z^{\prime}\right) \in \rho} y_{\rho}^{\prime}=\sum_{\left(\left(z \wedge z^{\prime}, z^{\prime}\right), D, 0, z\right) \in \rho} y_{\rho}^{\prime} .
$$

Now, simply set $n \in \mathbb{Z}_{+}^{\varrho}$ to be some multiple of $y^{\prime}$ large enough so that all coordinates are integer valued. We have

$$
\sum_{\left(\left(z \vee z^{\prime}, z\right), U, 0, z^{\prime}\right) \in \rho} n_{\rho}=\sum_{\left(\left(z \wedge z^{\prime}, z^{\prime}\right), D, 0, z^{\prime}\right) \in \rho} n_{\rho}
$$

and $\sum_{\rho \in \varrho} n_{\rho} \sum_{\left(\left(z, z^{\prime}\right), T, \lambda, z^{\prime}\right) \in \rho} \lambda \cdot\left(z-z^{\prime}\right)>0$. Thus the condition of cyclic supermodularity does not hold.

\section{References}

Afriat, S.: Efficiency estimation of production functions. Int Econ Rev 13(3), 568-598 (1972)

Afriat, S.N.: The construction of utility functions from expenditure data. Int Econ Rev 8(1), 67-77 (1967) Becker, G.S.: A theory of the allocation of time. Econ J 75(299), 493-517 (1965)

Berge, C.: Theory of Graphs. Dover Publications Inc. Mineola, New York (2001)

Brown, D.J., Calsamiglia, C.: The nonparametric approach to applied welfare analysis. Econ Theory 31(1), 183-188 (2007)

Chambers, C.P., Echenique, F.: Supermodularity is not falsifiable. Social Science Working Paper 1248. California Institute of Technology (2006)

Chung-Piaw, T., Vohra, R.: Afriat's Theorem and Negative Cycles. Mimeo, Northwestern University (2003)

Diewert, W.E., Parkan, C.: Linear progamming tests of regularity conditions for production functions. In: Eichhorn, W., Henn, R., Neumann, K., Shephard, R. (eds.) Quantitative Studies on Production and Prices, pp. 131-158. Vienna, Physica-Verlag (1983)

Fishburn, P.C.: The theorem of the alternative in social choice theory. Oper Res 19(6) (1971)

Fostel, A., Scarf, H., Todd, M.: Two new proofs of Afriat's Theorem. Econ Theory 24(1), 211-219 (2004)

Hanoch, G., Rothschild, M.: Testing the assumptions of production theory: a nonparametric approach. J Polit Econ 80(2), 256-275 (1972) 
Lancaster, K.J.: A new approach to consumer theory. J Polit Econ 74(2), 132-157 (1966)

Rockafellar, R.T.: Convex Analysis. Princeton: Princeton University Press (1970)

Topkis, D.M.: Supermodularity and Complementarity. Princeton: Princeton University Press (1998)

Varian, H.R.: The nonparametric approach to production analysis. Econometrica 52(3), 579-598 (1984)

Vives, X.: Oligopoly Pricing. Cambridge: MIT Press (1999) 